Canadian Oncology

Nursing Journal

Revue canadienne

de soins infirmiers

en oncologie

Volume 31, Issue 2 • Spring 2021

elSSN: 2368-8076 


\title{
Modèle de soins partagés pour les hémopathies malignes chroniques complexes
}

\author{
par Verna Cheung, Nancy Siddiq, Rebecca Devlin, Caroline McNamara et Vikas Gupta
}

\section{RÉSUMÉ}

Les néoplasies myéloprolifératives (NMP) sont des leucémies chroniques rares sans chromosome Philadelphie. L'expertise de cette maladie peu commune est limitée et concentrée dans des centres spécialisés où les patients doivent se rendre pour obtenir confirmation du diagnostic, prendre des décisions complexes, recevoir des soins de soutien et accéder à de nouveaux médicaments par la participation à des essais cliniques. Toutefois, les nombreux déplacements nécessaires pour ces rendez-vous représentent un poids pour les patients; ils augmentent le fardeau financier, accroissent le stress des proches aidants et nuisent à la qualité de vie. Pour s'attaquer à ce problème, le programme du Centre de cancérologie Princess Margaret (PM) dédié aux NMP a mis en place un modèle de soins partagés et a misé sur la collaboration avec les équipes soignantes locales afin d'offrir aux patients atteints de NMP une prise en charge continue et des soins de soutien plus accessibles. Ainsi, les patients se déplacent moins et reçoivent des soins de qualité axés sur leurs besoins. Le présent article décrit l'expérience de mise en ceuvre du modèle de soins partagés au Centre Princess Margaret, expérience qui pourrait s'étendre à d'autres hémopathies malignes chroniques et maladies chroniques rares. L'objectif ultime des soins partagés n'est pas de centraliser les services, mais plutôt de bâtir une communauté de soins accessibles pour le patient.

Mots clés : modèles de prestation des soins, leucémie chronique, NMP, soins partagés, hémopathies malignes

\section{AUTEURS \\ Verna Cheung, B.Sc.inf., inf. aut., M.Sc.inf., CSIO(C), infirmière clinicienne spécialisée, Centre de cancérologie Princess Margaret, Université de Toronto (Ontario)}

Nancy Siddiq, inf. aut., B.Sc.inf., CSIO(C), M.Sc.inf. en éducation, infirmière praticienne (adultes), Centre de cancérologie Princess Margaret, Université de Toronto (Ontario)

Rebecca Devlin, Ph.D., collaboratrice scientifique, programme des NMP Elizabeth and Tony Comper, Centre de cancérologie Princess Margaret, Université de Toronto (Ontario)

Caroline McNamara, MBBS (Hons), FRACP, FRCPA, MMed (épidémiologie clinique), hématologue titulaire, Centre de cancérologie Princess Margaret, Université de Toronto (Ontario)

Vikas Gupta, M.D., FRCP, FRCPath, professeur de médecine, Centre de cancérologie Princess Margaret, Université de Toronto (Ontario)

Auteur-ressource : Vikas Gupta, Centre de cancérologie Princess Margaret, 700 University Avenue, 6-326, Toronto (Ontario) M5G 175

Courriel : vikas.gupta@uhn.ca

DOI:10.5737/23688076312175185

\section{INTRODUCTION}

T e présent article a pour but de décrire le modèle de soins $\perp$ partagés adopté au Centre de cancérologie Princess Margaret (PM) pour les patients diagnostiqués d'une néoplasie myéloproliférative (NMP) sans chromosome Philadelphie. Ce modèle de soins partagés vise à mettre en place des soins de grande qualité dans lesquels les traitements sont coordonnés tant par le centre de cancérologie spécialisé que par les établissements de santé régionaux (Hershenfeld et al., 2017; Khera et al., 2017). Tout d'abord, nous décrirons les composantes du modèle de soins partagés du programme des NMP. Ensuite, nous illustrerons par des études de cas la mise en œuvre du modèle dans le programme des NMP du Centre PM, les leçons à tirer de cette expérience, les avantages et les défis du modèle, ainsi que le rôle de l'infirmière clinicienne spécialisée. L'article présentera les professionnels qui prodiguent les soins partagés : hématologues, oncologues et internistes des hôpitaux locaux. Les médecins de famille jouent également un rôle vital dans les soins, la prise en charge des comorbidités et les stratégies de prévention des risques cardiovasculaires. Cet article aborde principalement la gestion des NMP et des symptômes associés qui exigent une coordination entre les médecins des établissements de soins tertiaires et les hématologues, oncologues et internistes offrant des soins dans la collectivité.

\section{CONTEXTE}

Données probantes pour une bonne coordination des soins dans un modèle de soins partagés

Des recherches antérieures ont mis au jour les conséquences d'une mauvaise coordination : problèmes de communication, manque d'information pour les patients et proches aidants, lourdeur des déplacements et pression sur les proches aidants, absence de soutien psychosocial, financier et émotionnel, et visites fréquentes aux urgences (Gorin et al., 2017; Khera et al., 2017; Bazzell, Spurlock et McBride 2015).

La coordination des soins adopte de nombreuses formes, allant de la création d'un poste de coordonnateur ou d'intervenant pivot comme unique point de contact à la fois pour le patient et l'équipe interdisciplinaire à l'utilisation de technologies de l'information comme la télémédecine (Gorin et al., 2017; Khera et al., 2017; Chumbler et al., 2007; Girault et al., 2015). Dans un contexte de soins partagés, les études montrent que la coordination des soins améliore la qualité de vie, réduit le nombre d'hospitalisations non planifiées (Chumber et al., 2007), allège le fardeau des déplacements (Hershenfeld et al., 2017) et contribue à la compréhension et à la transmission des connaissances entre l'équipe spécialisée et les professionnels de la santé locaux (Nielsen et al., 2003). En retour, cette approche bâtit une communauté de pratique plutôt que de centraliser les soins, qui peuvent alors, pour la majeure partie, 
être offerts en toute sécurité à proximité du lieu de résidence des patients, augmentant leur satisfaction et améliorant leur qualité de vie générale (Gorin et al., 2017).

\section{Néoplasies myéloprolifératives}

Les NMP se caractérisent par la surproduction d'un ou plusieurs types de cellules sanguines matures (Arber et al., 2016). Il existe trois formes classiques de NMP sans chromosome Philadelphie : la polycythémie vraie (PV), la thrombocytémie essentielle (TE) et la myélofibrose primaire (MFP) (Mehta et al., 2014).

Dans le présent article, le terme « myélofibrose » (MF) englobe la MFP, la MF post-PV et la MF post-TE. Bien que les NMP classiques soient considérées comme des leucémies chroniques, la PV la TE et la MF se transformerait en leucémie myéloïde aiguë avec le temps (figure 1). Le risque d'évolution leucémique dépend de différents facteurs, comme le sous-type de NMP, l'âge et la présence d'anomalies génétiques à risque élevé comme des mutations cytogénétiques et somatiques (Mehta et al., 2014).

Chez les patients atteints de NMP, la surproduction d'un ou de plusieurs types de cellules sanguines augmente, les risques de complications médicales supplémentaires. Pour les patients souffrant de PV, l'incidence de complications hémorragiques est de $1,70 \%$ à $20 \%$, et chez ceux qui souffrent de TE, de 3,6 \% à $37 \%$ (Elliott et Tefferi, 2004). L'incidence d'accidents thrombotiques est de $12 \%$ à $39 \%$ dans les cas de PV, et de $11 \%$ à $25 \%$ dans les cas de TE (Elliott et Tefferi, 2004). L'embolie pulmonaire, l'accident vasculaire cérébral ou l'infarctus du myocarde font partie de ces complications hémorragiques et accidents thrombotiques qui détériorent la santé (Martin, 2017). La MF et la fibrose progressive de la moelle osseuse entravent l'hématopoièse, causant de l'anémie et une dépendance aux transfusions, ce qui se traduit par de fréquentes visites à l'hôpital pour recevoir des transfusions et par un risque accru de surcharge en fer (Mughal et al., 2014).

La MF peut également causer une hématopoìèse extramédullaire, qui entraîne une splénomégalie s'accompagnant de fortes douleurs abdominales, d'une satiété précoce et d'une diminution de l'appétit provoquant une importante perte de poids et de la cachexie (Mitra et al., 2013; Mughal et al., 2014). L'augmentation de la rate accroît la circulation sanguine dans le système porte, entraînant une hypertension portale qui, en retour, peut causer des varices œsophagiennes et gastriques (Mitra et al., 2013); l'anémie peut alors s'aggraver et exiger une intervention médicale. L'augmentation du débit sanguin dans le système porte peut aussi causer une thrombose et un infarctus splénique si l'apport sanguin dans la rate devient insuffisant (Mitra et al. 2013). D'autres symptômes pénibles comme une sudation nocturne importante, la fatigue et des douleurs généralisées touchent aussi communément les patients atteints de NMP, particulièrement dans les cas de MF (Harrison et al., 2017). Non seulement ces symptômes entravent la qualité de vie (Mughal et al., 2014), mais ils mettent à rude épreuve la relation entre le patient et les proches aidants (Harrison et al., 2017).

\section{NMP et modèle de soins partagés}

Les soins partagés se définissent comme « la collaboration entre les spécialistes et les professionnels des soins primaires et locaux dans le but d'offrir des soins planifiés aux patients souffrant de maladies chroniques, le tout s'appuyant sur une communication efficace » (Hall et al., 2011, p. 554-555). Le concept de soins partagés n'est pas nouveau; on l'utilise pour différentes maladies, notamment les hémopathies malignes, le diabète, les troubles mentaux et la toxicomanie (Hershenfeld et al., 2017; Khera et al., 2017; Smith et al., 2008). Grâce à ce modèle de soins, les patients ont accès à des traitements spécialisés tout en continuant de voir leur professionnel de la santé local pour recevoir les soins courants comme l'ajustement posologique, la prise en charge des comorbidités, le suivi de la numération globulaire et les transfusions sanguines d'entretien (Khera et al., 2017; Gorin et al., 2017).

Étant donné la complexité et la rareté des NMP, ce ne sont pas tous les hôpitaux locaux qui possèdent l'expertise ou l'expérience pour accueillir ces patients. Le Centre de cancérologie PM de Toronto (Canada) sert de ressource nationale vers laquelle sont dirigés les patients atteints de NMP. Grâce à ses capacités diagnostiques supérieures, le programme des NMP $\mathrm{du}$ Centre PM prête main-forte dans les cas difficiles en plus d'offrir beaucoup de traitements, dont l'allogreffe de moelle osseuse, ainsi que des essais cliniques et des soins de soutien à ce type de patients. Cependant, vu la nature complexe et chronique des NMP, le suivi et les soins de soutien sont nécessaires non seulement pour détecter et gérer les changements ou la progression de la maladie, mais aussi pour les autres comorbidités des patients afin de veiller à leur bien-être (Mehta et al., 2014).

Pour certains patients, se rendre au Centre PM est un véritable fardeau à cause de la distance, des coûts et d'autres difficultés (pression sur les proches aidants, organisation du transport, etc.). La création d'un partenariat de soins partagés avec les hôpitaux locaux atténue certains de ces problèmes (Hershenfeld et al., 2017). Les soins partagés facilitent également la transmission d'informations à l'équipe soignante locale et permettent aux patients de continuer à recevoir à l'hôpital local des soins tels que suivi de la numération globulaire, transfusions sanguines d'entretien en cas de besoin, renouvellement de la médication et ajustement posologique. En retour, les patients et les proches aidants passent moins de temps sur la route et leur quotidien s'en trouve moins bouleversé (Khera et al., 2017; Hershenfeld et al., 2017).

Des soins partagés réussis passent par une bonne coordination des soins (Khera et al., 2017; Chumbler et al., 2007) et une compréhension claire des attentes et responsabilités des principaux intervenants (figure 1). Dès le début, il importe également d'expliquer le modèle aux patients et aux proches aidants pour qu'ils comprennent le rôle de chaque professionnel dans les soins partagés.

La figure 1 illustre le modèle de soins partagés du Centre PM. Les patients, les familles, le Centre luimême (centre de cancérologie spécialisé), le fournisseur de soins partagés (hôpital local) et le coordonnateur des soins 
Figure 1. (A) Modèle de soins partagés pour les NMP

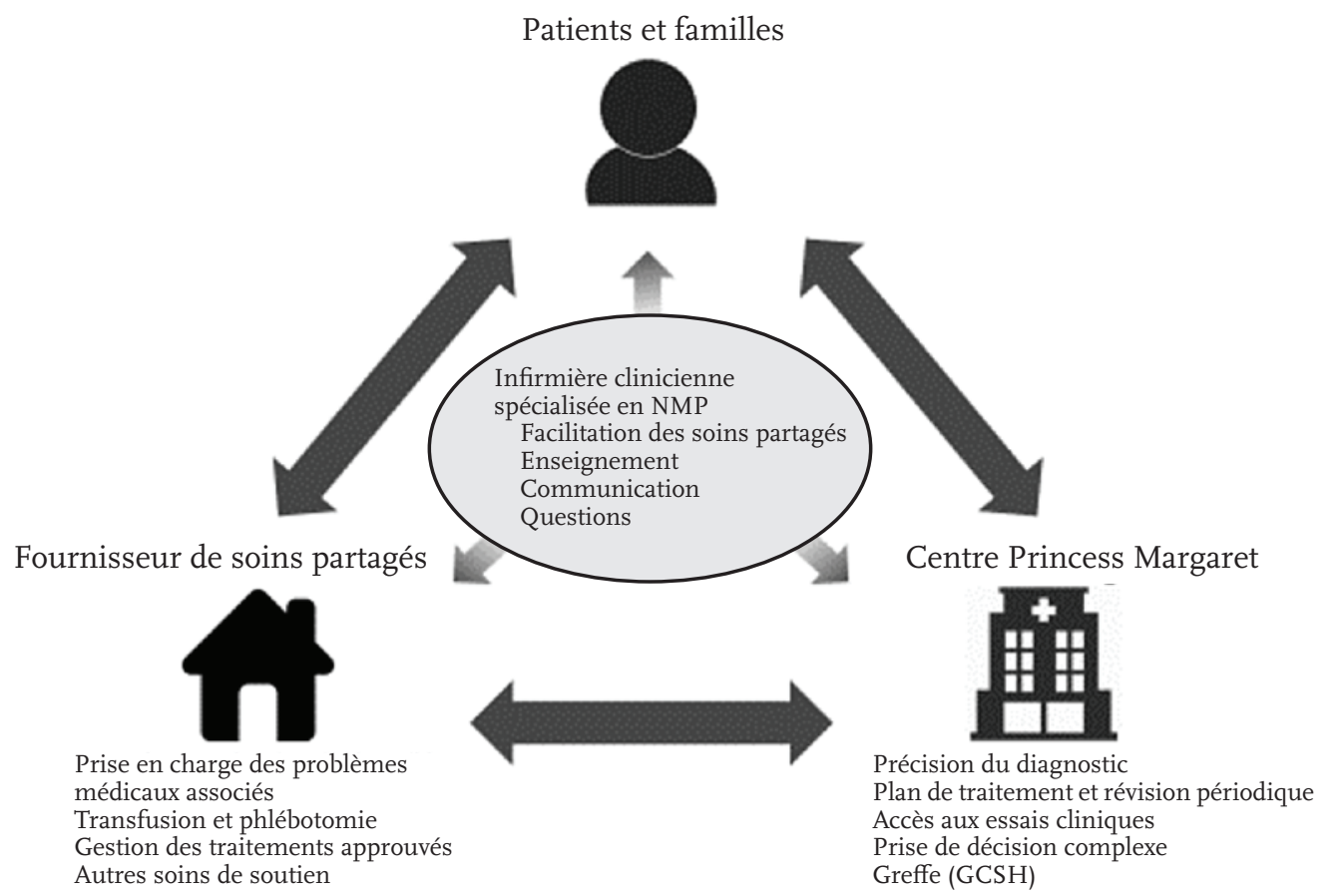

(B) déroulement du triage des patients atteints de NMP

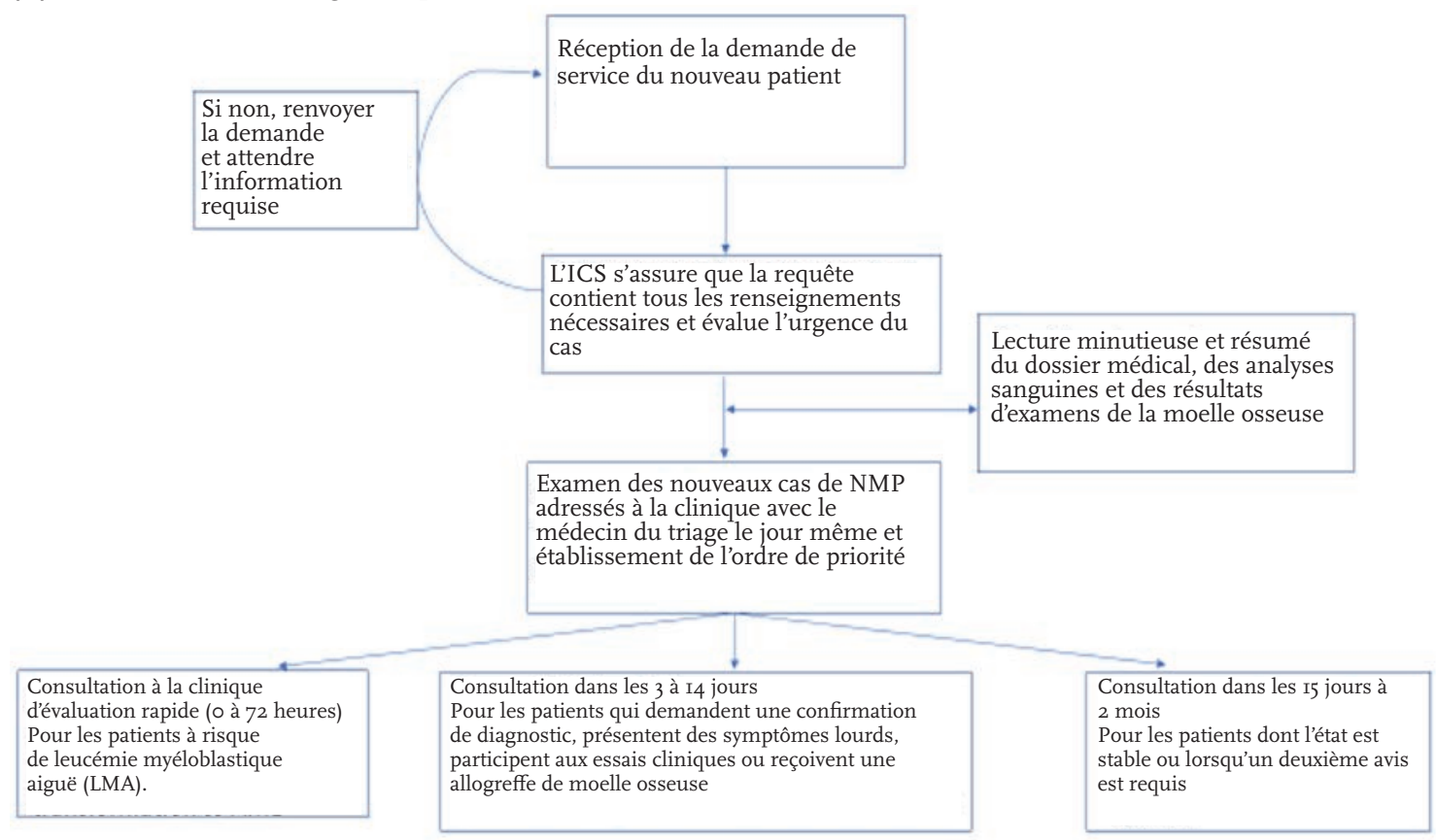

partagés sont les principaux intervenants du programme des NMP. Les responsabilités et les attentes pour chaque intervenant y sont clairement définies (Devlin et Siddiq, 2016; Tomasone et al., 2017). Ce programme confirme le diagnostic, donne des conseils sur les cas cliniques complexes, établit un plan de traitement, ouvre l'accès aux essais cliniques le cas échéant et participe à la prise de décision pour les traitements complexes comme l'allogreffe de cellules souches. Il confère également une autonomie accrue aux équipes soignantes locales en permettant l'accès à des ressources supplémentaires. De plus, l'équipe des NMP organise des rencontres annuelles avec les professionnels des soins partagés des différentes collectivités pour connaître leurs besoins en matière de formation et d'outils afin de faciliter leur participation au processus. De leur côté, les fournisseurs locaux de soins partagés prodiguent les traitements approuvés, de même que les soins de soutien continus (ex. suivi de la numération globulaire et transfusions d'entretien), en plus de surveiller et de gérer le traitement établi ainsi que les comorbidités (Devlin et Siddiq, 2016; Khera et al., 2017). Les patients doivent être 
vus aussi souvent que nécessaire, soit par le fournisseur local de soins partagés, soit par le Centre PM. Sous la direction de l'infirmière clinicienne spécialisée du programme des NMP, le coordonnateur des soins partagés collabore avec tous les intervenants pour favoriser la communication et l'organisation des soins, en plus de servir de ressources d'information sur la santé pour les patients et les proches aidants. Dans la figure 1, les flèches multidirectionnelles montrent la communication constante entre les membres de l'équipe de soins partagés.

Les patients adressés au programme des NMP du Centre PM sont souvent sous traitement intensif après avoir essayé sans succès un ou deux autres traitements; ils présentent également des comorbidités sous-jacentes qui compliquent la trajectoire de la maladie. Les études de cas qui suivent font ressortir la complexité clinique des cas de NMP et les bénéfices supplémentaires qu'apportent les soins partagés à ces patients.

\section{Première étude de cas}

La figure 2A montre le parcours de M. B., un homme de 58 ans ayant reçu un diagnostic de MF post-PV. À 53 ans, des prises de sang révélaient des taux élevés d'hémoglobine et d'hématocrite. Il a donc dû subir des examens hématologiques plus poussés. $\mathrm{Vu}$ la persistance de l'érythrocytose et la présence d'une mutation du gène $J A K 2$, on a considéré qu'il souffrait de PV et le traitement par phlébotomies a été initié.

Fait intéressant : un an plus tard, M. B. n'avait plus besoin de phlébotomies et présentait même une légère anémie. Avec le temps, il est malheureusement devenu fortement dépendant aux transfusions, il a développé une splénomégalie symptomatique et a manifesté des symptômes constitutionnels.

$\mathrm{Vu}$ le changement du tableau clinique de M. B., l'hématologue de l'hôpital local l'a dirigé vers le programme des NMP. Après avoir vu le patient, les spécialistes du programme ont confirmé le diagnostic de MF post-PV. Comme les symptômes étaient accablants, on l'a mis sous ruxolitinib et Eprex, une fois l'approbation obtenue pour cette médication. L'hématologue de l'hôpital local a effectué le suivi et l'ajustement posologique afin de réduire les déplacements du patient et, par le fait même, les perturbations de son quotidien.

Grâce aux soins partagés et à la communication soutenue entre l'hématologue de l'hôpital local et le programme des NMP, l'équipe soignante a constaté que, malgré l'amélioration des symptômes constitutionnels, M. B. présentait toujours une splénomégalie et qu'il devait se faire transfuser un concentré de globules rouges hebdomadairement. Il y avait aussi présence de blastes dans le sang périphérique, indice que la maladie demeurait très active. Lors d'un rendez-vous de suivi avec le programme des NMP du Centre PM, on a expliqué la situation à $\mathrm{M}$. B. et l'allogreffe de cellules souches a été envisagée. Malheureusement, l'équipe de greffe a dit qu'il fallait d'abord diminuer la splénomégalie avant de procéder à l'intervention.

À la suite de la recommandation de l'équipe de greffe, la posologie du ruxolitinib a été augmentée au maximum pour réduire l'hypertrophie de la rate et éviter la splénectomie. Léquipe des NMP a collaboré étroitement avec l'hématologue de l'hôpital local non seulement pour surveiller la numération globulaire et les transfusions d'entretien, mais aussi pour préparer $M$. B. à une éventuelle splénectomie si le ruxolitinib ne faisait pas diminuer suffisamment le volume de la rate en vue de la greffe. L'hématologue de l'hôpital local a organisé la vaccination au cas où $\mathrm{M}$. B. devrait subir une splénectomie et l'équipe des NMP lui a fixé un rendez-vous avec un chirurgien généraliste dans cette même optique.

Malheureusement, les doses élevées de ruxolitinib pendant six semaines n'ont pas eu les résultats escomptés. De concert, M. B. et l'équipe médicale ont donc décidé de procéder à une splénectomie; le programme des NMP, l'équipe de greffe, l'hématologue de l'hôpital local et le chirurgien généraliste étaient tous clairement au courant du plan de soins. Pendant tout le processus, le suivi n'a pas fait relâche; M. B. a pu faire ajuster la posologie de ruxolitinib, recevoir des transfusions sanguines d'entretien, se faire vacciner en prévision de la splénectomie et être confié rapidement à l'équipe de greffe après la splénectomie.

\section{Deuxième étude cas}

La situation clinique de M. P. est plus typique des cas adressés au programme de soins partagés des NMP. La figure $2 \mathrm{~B}$ illustre la trajectoire des soins de ce patient. À 25 ans, on lui a découvert un niveau de plaquettes élevé lors d'une simple prise de sang. Des examens hématologiques supplémentaires ont confirmé le diagnostic de TE.

$\mathrm{Au}$ fil des ans, M. P. a été suivi par un hématologue. Dans les trois dernières années, il a développé un carcinome papillaire au rein gauche et subi une néphrectomie totale. Il a été suivi en néphrologie (figure 2B). Peu de temps après, M. P. a présenté de l'essoufflement et a consulté aux urgences de l'hôpital local. On lui a alors diagnostiqué un épanchement péricardique nécessitant un drainage. Malheureusement, ces épisodes d'épanchement péricardique se sont produits de nouveau, obligeant une hospitalisation de M. P. Parallèlement, sa numération globulaire a chuté, aggravant la fatigue et les symptômes constitutionnels. Pendant son hospitalisation, les médecins de l'hôpital local ont procédé à une biopsie de la moelle osseuse, qui n'a pas révélé de progression vers la MF.

Lorsque nous avons reçu la demande de consultation de M. P. et pris en compte le fardeau des symptômes rapportés, la possible évolution en MF et les épanchements péricardiques récurrents, nous avons jugé qu'il était urgent qu'il vienne à la clinique et nous avons donc donné la priorité à son cas pour qu'il soit vu rapidement dans le cadre du programme des NMP.

Une consultation au programme des NMP du Centre PM et un examen coordonné des coupes de moelle osseuse ont confirmé le diagnostic de MF post-TE. L'analyse du liquide péricardique a également montré une hématopoï̀se extramédullaire. Le diagnostic confirmé, le patient a été mis sous traitement par inhibiteurs de JAK. Ces informations ont été communiquées à tous les professionnels de la santé de M. P. à l'aide de notes cliniques et de courriels pour qu'ils comprennent précisément les objectifs du traitement et le rôle de chaque discipline pour la poursuite des soins. L'hématologue 


\section{Figure 2A : Études de cas}

Homme de 58 ans, MF post-PV à mutation JAK2

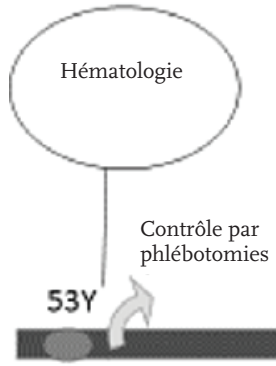

Numération globulaire élevée constatée lors d'une simple prise de sang

- Prurit aquagénique

- Détection de la

mutation JAK2

- PV présumée

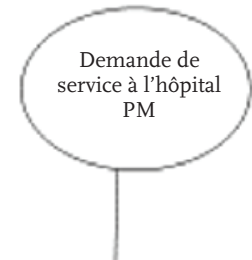

Anémie, mais Confirmation de sans dépendance aux transfusions

la MF post-PV par biopsi

de la moelle osseuse

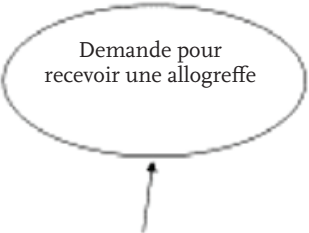

Diminution du volume de

la rate $(\mathrm{I} 9 \mathrm{~cm})$ qui demeure

hypertrophiée, sans symptômes

constitutionnels

Toujours dépendant aux transfusions

Blastes dans le sang

- Signe que la maladie est très active
Feu vert pour l'allogreffe

$56 Y$
Chute de l'hémoglobine

N'a plus besoin de phlébotomies mais bon état général

à 70

- Pas de saignement

- Dépendance aux
- Prurit toujours présent transfusions

- Splénomégalie $-27 \mathrm{~cm}$

- Perte de poids, sueurs

nocturnes

- Vérification de la progression de la maladie
Transfusions d'entretien administrées en soins partagés par l'hématologue de l'hôpital local pour limiter les perturbations du quotidien

L'EPREX n'ayant aucun effet, le traitement est interrompu
Début du traitement par La splénomégalie empêche la greffe.

Splénectomie inévitable.

Renvoi en chirurgie générale

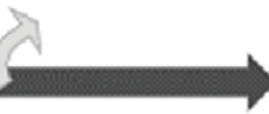


de l'hôpital local continue de surveiller et de gérer la NMP, ce qui diminue le nombre de visites au Centre PM. Le patient passe donc moins de temps en déplacement, son quotidien s'en trouve moins perturbé et la personne l'accompagnant aux rendez-vous voit son fardeau allégé. Le son de cloche de l'équipe de cardiologie sur la situation clinique de M. P. a permis d'éviter des interventions cardiaques invasives et cette équipe continuera de participer au suivi pour surveiller l'état cardiovasculaire du patient et optimiser les soins.

\section{Résumé}

Les deux études de cas illustrent la complexité des NMP, non seulement de la maladie en tant que telle, mais aussi des comorbidités associées, du nombre de spécialités médicales concernées et le risqué élevé de fragmentation des soins, qui peuvent vite devenir accablants pour le patient. Lorsqu'une bonne coordination est en place, les soins partagés permettent au patient atteint de NMP de recevoir les soins spécialisés dont il a besoin et qui ne sont pas toujours offerts au centre de cancérologie local; il peut aussi obtenir confirmation du diagnostic et accéder aux essais cliniques ou aux nouveaux traitements tout en recevant des soins de soutien au centre de cancérologie de sa région. Le coordonnateur des soins veille à communiquer le plan de traitement et les prochaines étapes de soins à tous les membres de l'équipe soignante, assurant ainsi la cohésion des interventions.

\section{NMP et modèle de soins partagés : leçons à tirer}

Le programme des NMP du Centre PM a mis en place le modèle de soins partagés en 2014. Pour que ce modèle ait le succès escompté, il a besoin de ressources dédiées, notamment d'un point de contact unique, d'une bonne coordination des soins, de moyens pour garder la communication ouverte et d'attentes claires quant aux rôles et responsabilités des différents professionnels de la santé. Le tableau 1 résume brièvement les besoins ainsi que les solutions efficaces du point de vue de l'expérience acquise au Centre PM.

Pour que les communications soient claires, cohérentes et rapides, et que la coordination des soins soit adéquate, le modèle de soins partagés du programme des NMP emploie une infirmière clinicienne spécialisée (ICS) pour faciliter le processus. L'ICS détient une maîtrise et possède des compétences avancées en évaluation clinique, ainsi qu'une connaissance approfondie des NMP; de plus, l'ICS prend part à tous les aspects des soins aux patients et travaille en collaboration avec l'équipe de médecins spécialistes des NMP. Dans son rôle, elle voit au triage des nouveaux patients adressés au Centre, participe à la première consultation, ainsi qu'au suivi des patients à la clinique, et donne de l'enseignement aux patients et aux proches aidants sur le diagnostic, les pistes de traitement, les soins de soutien, la planification familiale et le contrôle des symptômes. L'ICS travaille aussi avec les patients pour faciliter et coordonner les soins qui seront prodigués localement. De plus, étant donné le vaste champ de pratique de l'ICS, elle sert aussi de personne-ressource et de point de contact pour les patients et les proches aidants, ainsi que pour les infirmières des établissements de soins locaux lorsqu'elles doivent clarifier certains points ou qu'elles ont des questions
Tableau 1 : Leçons à tirer pour assurer le succès des soins partagé

\begin{tabular}{|l|l|}
\hline Besoins & $\begin{array}{l}\text { Solutions pour assurer la réussite des soins } \\
\text { partagés }\end{array}$ \\
\hline Ressources & $\begin{array}{l}\text { - Capacité clinique d'accueillir les patients } \\
\text { atteints de NMP } \\
\text { - Membres spécialisés de l'équipe } \\
\text { interdisciplinaire (ex. médecins, ICS, etc.) } \\
\text { qui voient le patient et gèrent les soins } \\
\text { - Outils d'évaluation appropriés pour mesurer } \\
\text { les symptômes (version numérique du } \\
\text { questionnaire sur les symptômes de NMP } \\
\text { pour suivre l'évolution des symptômes) }\end{array}$ \\
\hline
\end{tabular}

Point de contact/ - ICS au Centre PM dont le travail est dédié membre de au programme des NMP et aux patients l'équipe dédié à atteints de NMP

cette tâche $\quad$ - Le point de contact peut également être une infirmière pivot, un coordonnateur, etc., mais il doit se consacrer à ce groupe particulier de patients.

Communication - Membre dédié de l'équipe (ex. ICS du claire, concise et programme des NMP du Centre PM) rapide entre tous ayant la capacité de participer à toute les intervenants la trajectoire des soins du patient en collaborant avec tous les intervenants pour assurer une communication claire, concise et rapide.

- L'infirmière clinique responsable de la ligne de triage apporte un soutien supplémentaire.

Acceptation des - Communication claire des attentes des soins partagés par les patients et les soins partagés et de leurs avantages

proches aidants

\begin{tabular}{|c|c|}
\hline $\begin{array}{l}\text { Assentiment } \\
\text { et capacité des } \\
\text { partenaires } \\
\text { communautaires } \\
\text { (ressources et } \\
\text { expertise du } \\
\text { personnel) }\end{array}$ & $\begin{array}{l}\text { - Communication claire des attentes et des } \\
\text { responsabilités, ainsi que des avantages des } \\
\text { soins partagés. } \\
\text { - Disponibilité en tout temps pour les } \\
\text { partenaires communautaires afin de } \\
\text { répondre à leurs préoccupations. } \\
\text { - Transfert des connaissances : ressources } \\
\text { à jour, ex. séances de formation pour les } \\
\text { partenaires locaux, MPN eSIMPLE. }\end{array}$ \\
\hline $\begin{array}{l}\text { Normalisation: } \\
\text { protocole de } \\
\text { traitement, } \\
\text { demande } \\
\text { de service, } \\
\text { documentation } \\
\text { destinée aux } \\
\text { patients }\end{array}$ & $\begin{array}{l}\text { - Accès à de la documentation créée par } \\
\text { l'équipe des NMP pour les patients en guise } \\
\text { de ressource } \\
\text { - Formulaires normalisés : demande de } \\
\text { service, triage (pour établir l'ordre de } \\
\text { priorité), définition précise des rôles et } \\
\text { responsabilités }\end{array}$ \\
\hline $\begin{array}{l}\text { Soutien } \\
\text { psychosocial et } \\
\text { financier }\end{array}$ & $\begin{array}{l}\text { - Travailleur social, spécialiste du } \\
\text { remboursement des frais médicaux, } \\
\text { oncologie psychosociale et programme de } \\
\text { soins palliatifs pour aider les patients et les } \\
\text { jumeler avec les ressources existantes dans } \\
\text { leur collectivité }\end{array}$ \\
\hline
\end{tabular}


sur la prise en charge propre à la NMP, les symptômes, les médicaments et leurs effets secondaires. Les cliniques d'hémopathies malignes du Centre PM disposent également d'une ligne téléphonique de triage où une infirmière apporte une aide supplémentaire pour le contrôle des symptômes et le renouvellement de la médication.

Vu son champ de pratique étendu, son niveau de participation aux soins des patients et le temps qu'elle consacre au programme des NMP, l'ICS est bien placée pour servir de point de contact unique (Khera et al., 2017), mettre le patient en lien avec les professionnels de la santé et s'assurer que toute l'équipe multidisciplinaire à l'extérieur du Centre PM reçoit l'information.

Il essentiel d'établir des lignes directrices claires pour les soins partagés, notamment les attentes et les responsabilités des équipes locales, et de bien définir les avantages du modèle dans la cogestion des soins aux patients. Le programme des NMP a normalisé les processus d'aiguillage et de triage pour assurer l'efficacité et l'exactitude de la hiérarchisation des patients orientés vers le programme (Khera et al., 2017; Tomasone et al., 2017). La figure 3 illustre l'organisation générale du triage dans le programme des NMP, qui prévoit notamment la mise en œuvre d'un guide pour le triage et l'aiguillage. Le formulaire d'orientation vers les services explique les processus normalisés aux fournisseurs de soins partagés pour qu'ils leur communiquent toute l'information clinique pertinente, ce qui facilite le triage. Les établissements ayant fait une demande de consultation pour leur patient reçoivent une lettre confirmant son admission au programme et décrivant les attentes quant aux soins partagés (figures supplémentaires 1 et 2).

Comme les patients et les proches aidants sont des figures clés des soins partagés, ils doivent impérativement comprendre la valeur et les avantages de cette approche. Au moment de fixer le premier rendez-vous avec le patient, on lui expose clairement les attentes des soins partagés.

Le transfert des connaissances (Nielsen et al., 2003) et l'accès aux ressources nécessaires pour les partenaires de soins partagés sont des étapes essentielles pour maintenir la prestation des soins, garantir des soins de soutien aux patients atteints de NMP (Khera et al., 2017), et bâtir la confiance de ces derniers envers leur équipe soignante locale. Le transfert des connaissances normalise également les pratiques et augmente la confiance des patients envers les soins partagés. Le programme des NMP du Centre PM recourt à différentes méthodes pour transmettre les connaissances aux partenaires des soins partagés. Il offre, par exemple, des séances de formation et de perfectionnement en cours d'emploi à l'équipe soignante locale pour exposer les derniers développements dans la prise en charge des NMP. À cette occasion, l'équipe locale est invitée à présenter le cas de NMP dont elle souhaite discuter du diagnostic, du traitement et de la prise en charge. De plus, les partenaires locaux de soins partagés peuvent joindre les membres de l'équipe des NMP par courriel et par téléphone pour discuter de la prise en charge d'un patient suivi en soins partagés ou de nouveaux cas complexes qu'ils rencontrent. Les notes cliniques permettent aussi de transférer des connaissances et de communiquer en continu l'information sur les patients suivis en soins partagés. Tant l'équipe des NMP et que le partenaire local des soins partagés doivent être au courant du plan de soins.

Le programme des NMP utilise aussi un autre mode de transfert des connaissances : l'application MPN eSIMPLE, lancée en 2016. Il s'agit d'une initiative de collaboration entre le Canadian MPN Group et Novartis. L'application est accessible à partir d'un site Web; il existe également une version mobile. L'application MPN eSIMPLE renseigne les partenaires locaux de soins partagés, les patients et les proches aidants sur les possibilités de traitement de la PV, de la TE et de la MF, et met à leur disposition des calculatrices de stratification du risque. La figure supplémentaire 3 montre la consultation du site à l'échelle nationale et internationale.

\section{ÉVALUATION ET CONCLUSION}

L'efficacité de chaque modèle et programme doit être évaluée régulièrement (Khera et al., 2017). En analysant les succès et les avantages du modèle de soins partagés, ainsi que les points à améliorer, les intervenants sont plus à même d'en constater l'efficacité (Khera et al., 2017; Schultz et al., 2013; McWilliam, 2016). Les facteurs tels que la diminution des coûts, des déplacements et du recours aux soins de santé (ex. hospitalisations, visites aux urgences) ne sont pas les seuls paramètres à observer; l'expérience des patients, leur sécurité et leur satisfaction envers les soins, la perturbation de leurs activités quotidiennes, de même que le changement des habitudes et des connaissances en matière de santé, doivent aussi entrer en ligne de compte (McWilliam, 2016; Hershenfeld et al., 2017).

Ces mêmes paramètres seront également utiles pour évaluer le modèle de soins partagés du programme des NMP, par exemple à l'aide d'un questionnaire. Le programme travaille à la mise au point d'un outil d'évaluation pour comprendre l'expérience des soins partagés vécue par les patients et les proches aidants. L'information recueillie permettra à l'équipe de cerner les points positifs et les domaines à améliorer puis d'orienter les projets à venir.

Les NMP sont des cancers chroniques rares qui évoluent parfois en leucémie aiguë. L'adoption d'un modèle de soins partagés aux interventions bien coordonnées facilite le suivi des patients par les équipes soignantes locales et les équipes spécialisées en oncologie, ce qui assure une prise en charge efficace de la maladie et permet de modifier le traitement si l'état du patient se détériore. D’autres maladies chroniques rares pourraient également bénéficier de ce modèle de soins collaboratifs de grande qualité.

\section{REMERCIEMENTS}

Nous aimerions remercier toutes les infirmières responsables des patients leucémiques à la clinique ambulatoire pour leur précieux soutien tant en clinique qu'à la ligne de triage. 
Figure supplémentaire 1 : Formulaire de demande de service au programme des NMP

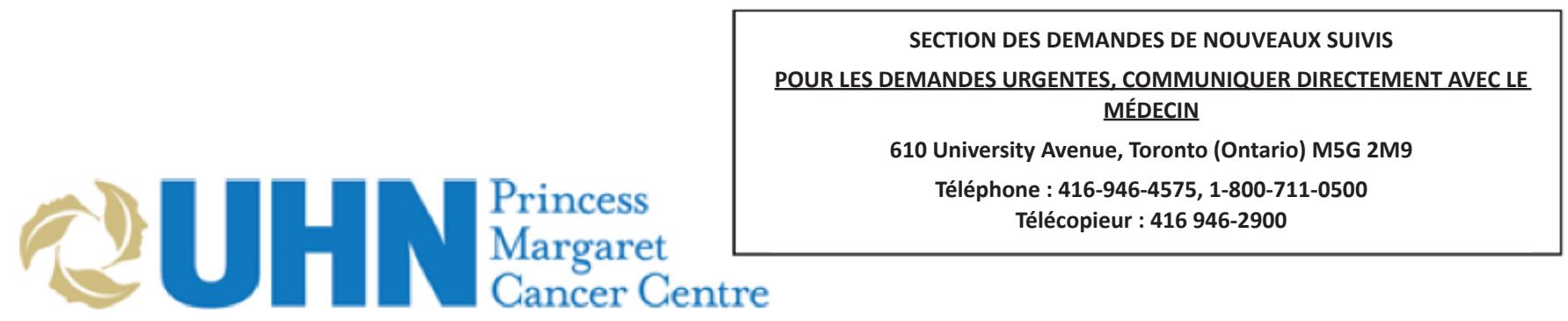

Date d'envoi :

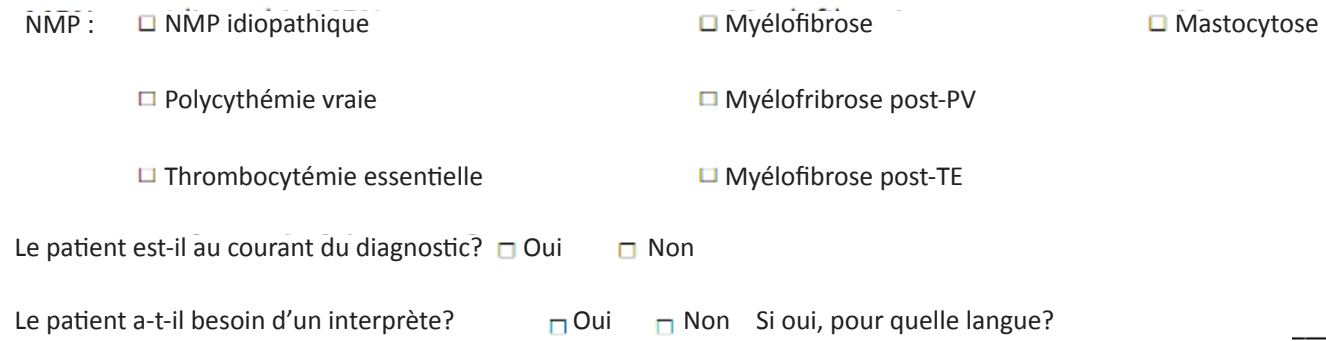

Le patient est-il áu courant du diagnostic? $\square$ Oui $\square$ Non

Le patient a-t-il besoin d'un interprète? $\quad \square$ Oui $\quad \square$ Non Si oui, pour quelle langue?

*INFORMATION CLINIQUE REQUISE* (Veuillez donner le plus d'information possible et envoyer par TÉLÉCOPIEUR TOUS LES RAPPORTS DE CONSULTATION ET LES NOTES CLINIQUES.)

\begin{tabular}{|l}
\hline Motif de consultation : \\
$\square$ Diagnostic récent \\
$\square$ Deuxième avis \\
$\square$ Maladie récurrente/évolutive \\
$\square$ Essais cliniques \\
$\square$ Consultation en vue d'une allogreffe de moelle \\
osseuse
\end{tabular}

Diagnostic

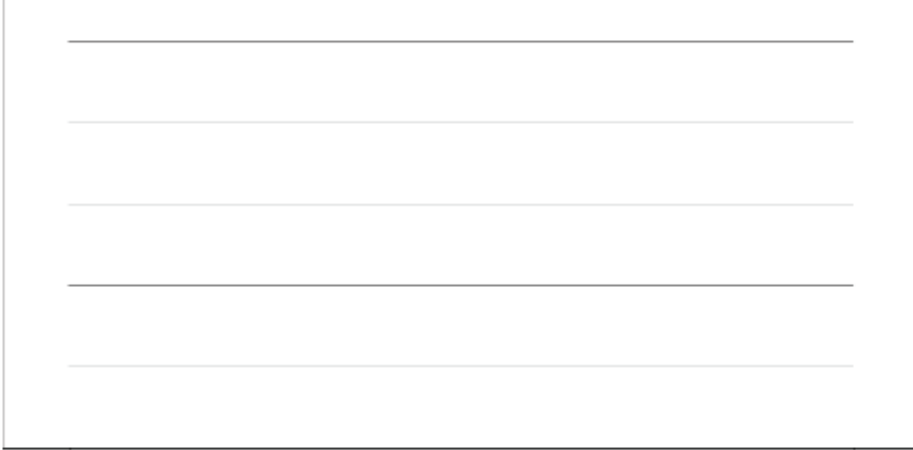

\section{VEUILLEZ JOINDRE LES ÉLÉMENTS SUIVANTS LE CAS ÉCHÉANT :}

- Profil démographique du patient

- Notes cliniques

- Résultats des analyses de sang : FSC, profil biochimique

- Vitamine B12 et ferritine (si vérifié)
- Résultats de l'aspiration et de la biopsie de moelle osseuse

- Cytogénétique ( $\square$ fait $\square$ en cours $\square$ non vérifié)

- Analyses moléculaires

$\square$ JAK2 $\square$ CALR $\square$ BCR-ABL

\section{NOTE : LE SUIVI DU PATIENT DEMEURE SOUS LA RESPONSABILITÉ DE SON MÉDECIN TRAITANT, MÊME APRÈS LA} CONSULTATION AU CENTRE. 
Figure supplémentaire 2 : Formulaire de triage des patients atteints de NMP

Formulaire de triage des nouveaux patients atteints de NMP

\begin{tabular}{|c|c|c|c|c|}
\hline Date de réception de la demande: & \multicolumn{2}{|c|}{ Nom du patient } & & \\
\hline Date de vérification: & \multicolumn{2}{|c|}{ No dossier médical } & & \\
\hline Par (nom du médecin) : & \multicolumn{2}{|c|}{ À voir dans } & & \\
\hline \multicolumn{2}{|l|}{$\begin{array}{l}\text { Le patient devrait-il être vu en clinique } \\
\text { d'évaluation rapide? }(\mathrm{V} / \mathrm{x})\end{array}$} & \multicolumn{3}{|c|}{ Niveau de priorité } \\
\hline \multicolumn{2}{|c|}{$\begin{array}{l}\text { Les patients qui répondent à un critère ou plus doivent } \\
\text { être vus en clinique d'évaluation rapide. }\end{array}$} & \multirow[t]{2}{*}{$\begin{array}{l}\text { Niveau } 1 \\
0 \text { à } 72 \mathrm{~h}\end{array}$} & \multirow[t]{2}{*}{$\begin{array}{l}\text { Niveau } 2 \\
3 \text { à } 14 \text { jours }\end{array}$} & \multirow[t]{2}{*}{$\begin{array}{l}\text { Niveau } 3 \\
15 \text { jours }<2 \text { mois }\end{array}$} \\
\hline \multicolumn{2}{|c|}{ - $\quad$ plaquettes $<50$} & & & \\
\hline \multicolumn{2}{|l|}{ - $\quad$ neutropénie<0,5 } & & & \\
\hline \multicolumn{2}{|l|}{$\begin{array}{l}\text { - Augmentation des blastes dans le sang } \\
\text { périphérique / moelle osseuse }>10 \%\end{array}$} & & & \\
\hline - Transformation blastique suspectée & & & & \\
\hline
\end{tabular}

\begin{tabular}{|l|l|}
\hline 1. Ce patient a-t-il été accepté à la clinique des NMP? Oui/Non & 2 . Essais cliniques? Oui/Non \\
\hline
\end{tabular}

3. Motif principal de la demande:

4. Résumé clinique

5. Des typages HLA ou PRA sont-ils requis? Oui/Non Si oui, pourquoi?

\begin{tabular}{|c|c|c|c|c|}
\hline \multicolumn{5}{|c|}{ Analyses sanguines: } \\
\hline EPO : & AST : & HGB : & G. BLANCS : & Moelle Osseuse: \\
\hline Créatinine: & PALC: & $\mathrm{HCT}$ : & Neutrophiles: & Aspiration \\
\hline $\mathrm{LDH}:$ & ALT: & VGM: & & Biopsie \\
\hline Ferritine: & Réticulocytes: & PLAQ : & & \\
\hline
\end{tabular}

\begin{tabular}{|l|l|l|l|}
\hline Cytogénétique (encercler la réponse) : Normale Anormale Non concluante $\quad$ Non vérifiée/S.O. \\
\hline Mutation (encercler tous les éléments qui s'appliquent) : & MPL & BCR-ABL \\
\hline JAK2: V617F EXON 12 & CALR & \\
\hline Autres
\end{tabular}

\begin{tabular}{|l|l|}
\hline \multicolumn{2}{|l|}{ Considérations particulières: } \\
\hline Accessibilité: & \\
\hline Interprète: & Langue: \\
\hline
\end{tabular}

Date de rendez-vous à la clinique : 
(A) Au Canada

\section{Consultation par ville (\%)}

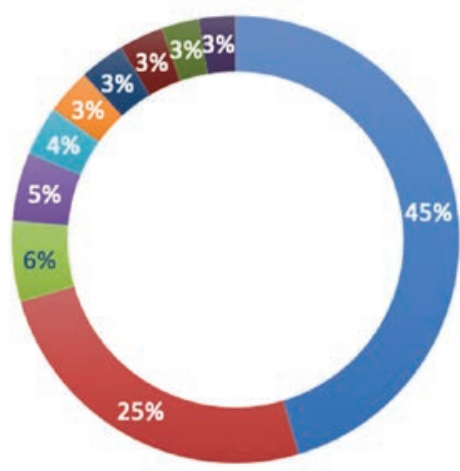

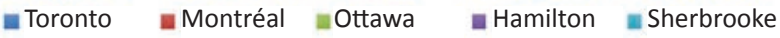

= London $\quad$ Laval $\quad$ Mississauga $=$ Vancouver $=$ Calgary

\section{(B) à l'international}

\section{Consultation du site par pays (\%)}

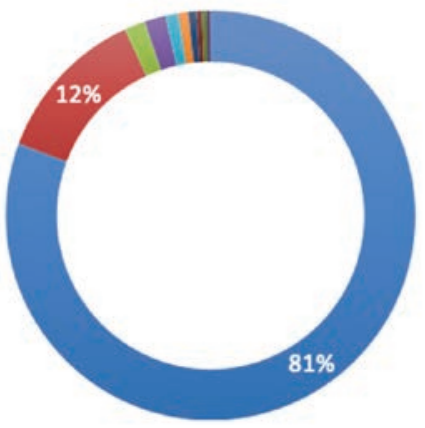

\begin{tabular}{|c|c|c|c|}
\hline - Canada & États-Unis & Australie & - Italie \\
\hline Brézil & Allemagne & - Colombie & a France \\
\hline E Iran & —Suisse & & \\
\hline
\end{tabular}

\section{RÉFÉRENCES}

Arber, D. A., Orazi, A., Hasserjian, R., Thiele, J., Borowitz, M. J., Le Beau, M. M., Bloomfield, C. D., Cazzola, M. \& Vardiman, J. W. (2016). The 2016 revision to the World Health Organization classification of myeloid neoplasm and acute leukemia. Blood, 127(20), 2391-2405.

Bazzell, J. L., Spurlock, A., \& McBride, M. (2015). Matching the unmet needs of cancer survivors to resources using a shared care model. Journal of Cancer Education, 30, 312-218.

Chumbler, N. R., Kobb, R., Harris, L., Richardson, L. C., Darkins, A., Sberna, M., Dixit, N., Ryan, P., Donaldson, M., \& Krebs, G. L. (2007). Healthcare utilization among veternas undergoing cChemotherapy - The impact of a cancer care coordination/hometelehealth program. Journal of Ambulatory Care Managment, 30(4), 308-317.

Devlin, R. \& Siddiq, N. M. (2016). Myeloproliferative Neoplasms (MPN): A guide for patients a families. University Health Network, Toronto.

Elliott, M. A., \& Tefferi, A. (2004). Thrombosis and haemorrhage in polycythemia vera and essential thrombocythaemia. British Journal of Haematology, 128, 275-290.

Girault, A., Ferrua, M., Lalloue, B., Sicotte, C., Fourcade, A., Yatim, F., Hebert, G., Di Palma, M., \& Minivielle, E. (2015). Internet-based technologies to improve cancer care coordination: Current use and attitudes among cancer patients. European Journal of Cancer, 51, 551-557.

Gorin, S. S., Haggstrom., D., Han, P. K. J., Fairfield, K. M., Krebs, P., \& Clauser, S. B. (2017). Cancer care coordination: A systematic review and meta-analysis of over 30 years of empirical studies. Annals of Behavioral Medicine, 51(4), 532-546.

Harrison, C. N., Koschmieder, S., Foltz, L., Guglielmelli, P., Flindt, T., Koehler, M., Mathias, J., Komatsu, N., Boothroyd, R. N., Spierer, A., Ronco, J. P., Taylor-Stokes, G., Waller, J., \& Mesa, R. A. (2017). The impact of myeloproliferative neoplasm (MPN) on patient quality of life and productivity: results from the international MPN Landmark survey. Annals of Hematology, 96(10), 1653-1665.

Hershenfeld, S. A., Maki, K., Rothfels, L., Murray, C. S., Nixon, S., Schimmer, A. D., \& Doherty, M. C. (2017). Sharing post-AML consolidation supportive therapy with local centres reduces patient travel burden without compromising outcomes. Leukemia Research, 50, 93-96.

Khera, N., Martin, P., Edsall, K., Bonagura, A., Burns, L. J., Juckett, M., King, O., LeMaistre, C. F., \& Majhail, N. S. (2017). Patientcentered care coordination in hematopoietic cell transplantation. Blood advances, 1(19), 1617-1627. https://doi.org/10.1182/ bloodadvances.2017008789

Martin, K. (2017). Risk factors for and management of MPN-associated bleeding and thrombosis. Current Hematologic Malignancy Reports, 12(5), 389-396.

McWilliams, J. M. (2016). Cost containment and the tale of care coordination, New England Journal of Medicine, 375(23), 2218-2220.

Mehta, J., Wang, H., Iqbal, S. U., \& Mesa, R. (2014). Epidemiology of myeloproliferative neoplasms in the United States. Leukemia Q Lymphoma, 55(3), 595-600.

Mitra, D., Kaye, J. A., Piecoro, L. T., Brown, J., Reith, K., Mughal, T. I., \& Sarlis, N. J. (2013). Symptom burden and splenomegaly in patients with myelofibrosis in the United States: A retrospective medical record review. Cancer Medicine, 2(6), 889-898.

Mughal, T. I., Vaddi, K., Sarlis, N. J., \& Verstovsek., S. (2014) Myelofibrosis-associated complications: Pathogenesis, clinical manifestations, and effects on outcomes. International Journal of General Medicine, 7, 89-101.

Nielsen, J. D., Palshof, T., Mainz, J., Jensen, A. B., \& Olesen, F. (2003). Randomized controlled trials of a shared care programme for newly referred cancer patients: bridging the gap between general practice and hospital. Quality \& Safety in Health Care, 12 (4), 263-272.

Schultz, E. M., Pineda, N., Lonhart, J., Davies, S. M., \& McDonald, K. M. (2013). A systematic review of the care coordination measurement landscape. BMC Health Service Research, 13(119), $1-12$.

Smith, S. M., Allwright, S., \& O’Dowd, T. (2008) Does sharing care across the primary-speciality interface improve outcomes in 
chronic diseases? A systematic review. American Journal of Managed Care, 14, 213-224.

Tomasone, J. R., Vukmirovic, M., Brouwers, M. C., Grunfeld, E., Urquhart, R., O’Brien, M. A., Walker, M., Webster, F., \& Fitch, M. (2017). Challenges and insights in implementing coordinated care between oncology and primary care providers: A Canadian perspective. Current Oncology, 24(2), 120-123. 